\title{
Interacting entropy-corrected holographic Chaplygin gas model
}

\author{
M. Umar Farooq,,$*$ Muneer A. Rashid, ${ }^{1}$ and Mubasher Jamil ${ }^{1,+}$ \\ ${ }^{1}$ Center for Advanced Mathematics and Physics, \\ National University of Sciences and Technology, Rawalpindi, 46000, Pakistan
}

\begin{abstract}
Holographic dark energy (HDE), presents a dynamical view of dark energy which is consistent with the observational data and has a solid theoretical background. Its definition follows from the entropy-area relation $S(A)$, where $S$ and $A$ are entropy and area respectively. In the framework of loop quantum gravity, a modified definition of HDE called "entropy-corrected holographic dark energy" (ECHDE) has been proposed recently to explain dark energy with the help of quantum corrections to the entropy-area relation. Using this new definition, we establish a correspondence between modified variable Chaplygin gas, new modified Chaplygin gas and the viscous generalized Chaplygin gas with the entropy corrected holographic dark energy and reconstruct the corresponding scalar potentials which describe the dynamics of the scalar field.
\end{abstract}

*Electronic address: mfarooq@camp.nust.edu.pk

$\dagger$ Electronic address: mjamil@camp.edu.pk 


\section{INTRODUCTION}

Numerous cosmological observations of type Ia supernova, cosmic microwave background anisotropies measured with WMAP and large scale structure, suggest that our universe is undergoing in an accelerated expansion possibly due to the presence of dark energy which possesses negative pressure [1]. In the astrophysics community, the nature of such mysterious form of energy is still a matter of debate. In cosmology, several candidates responsible for this expansion have been proposed namely, Chaplygin gas, modified gravity, scalar tensor theory, tachyon and braneworld model, to a name a few [2]. Despite the fact that the cosmological constant is the most obvious candidate that offers a solution to the dark energy problem, yet it has several drawbacks like fine-tuning and coincidence problems.

In order to soften the cosmic coincidence and fine tuning problems, models of dark energy interacting with dark matter have been proposed [3]. It is generally asked that if the universe evolves from the earlier quintessence $(\omega>-1)$ to late accelerating universe with phantom regime $(\omega<-1)$, then why $\omega=-1$ crossing occurs at the present time, where $\omega=p / \rho$. The equation of state (EoS) parameter $\omega$ changes at different cosmic epoch which supports the evolving dark energy [4]. In the cosmological model explaining dark energy, the ratio of energy densities of dark matter and dark energy $r_{m}$ is of order unity, while observations suggest that in pure dark energy model, this ratio must decrease. It may implies the transfer of energy occurs between these two entities to keep a subtle balance at the current time. The interaction between these entities involves a coupling constant that determines the strength of this interaction. Besides Einstein gravity, this study of interaction can also be extended to $f(R)$, Brans-Dicke, braneworld, Horava-Lifshitz and Gauss-Bonnet gravities [5].

In recent times, considerable interest has been stimulated to explain the observed dark energy (dominant force in cosmos) with the help of holographic dark energy model [6]. According to holographic principle, the number of degrees of freedom in a bounded physical system should be finite and has relationship with the area of its boundary rather than with its volume [7]. It is commonly believed that the holographic principle is a fundamental principle of quantum gravity which is used to explain the events involving high energy scale. It is motivated from an observation that in quantum field theory, the ultra-violet cut-off $\Lambda$ could be related to the infrared cut-off $L$ due to the limit set by forming a black hole i.e. the quantum zero-point energy of a system with size $L$ should not exceed the mass of a black 
hole with the same size, i.e. $L^{3} \Lambda^{3} \leq\left(M_{p} L\right)^{3 / 2}[8]$. This last expression can be re-written as $L^{3} \rho_{\Lambda} \leq L M_{p}^{2}$, where $\rho_{\Lambda} \backsim \Lambda^{4}$ is the energy density corresponding to the zero point energy and cut-off $\Lambda$. Now the last inequality takes the form $\rho_{\Lambda} \leq M_{p}^{2} L^{-2}$ or $\rho_{\Lambda}=3 n^{2} M_{p}^{2} L^{-2}$. Here $3 n^{2}$ is a constant and attached for convenience. A sufficient literature is available on the study of interaction between the holographic dark energy and the matter [9]. We are interested in studying the dynamics of entropy corrected holographic dark energy (ECHDE) when it interacts with some exotic type fluids. The entropy corrected holographic dark energy is given by [10]

$$
\rho_{\Lambda}=3 n^{2} m_{p}^{2} L^{-2}+\gamma L^{-4} \ln \left(m_{p}^{2} L^{2}\right)+\beta L^{-4},
$$

where the first term on right hand side is the usual holographic dark energy while the other two appeared are mainly due to quantum corrections in the loop quantum gravity (LQG). The correction term play crucial role in early universe when $\mathrm{L}$ is small. When $\mathrm{L}$ gets large, ECHDE reduces to HDE. The expression for the corrected entropy of the black hole is given by $S=\frac{A}{4 G}+\gamma \ln \left(\frac{A}{4 G}\right)+\beta$ is mainly arise due to the thermal equilibrium and quantum fluctuation [11]. The parameters $n^{2}, \gamma$ and $\beta$ constant of order unity. If we choose $\gamma$ and $\beta$ to be zero we arrive at the usual holographic dark energy model. The parameter $n$ can be a function of time [12], but in our discussion it is purely a constant quantity.

The plane of the paper is as follows: In section 2, the model of Friedmann-RobertsonWalker (FRW) universe and the relevant equations are presented. In section 3, we discuss a correspondence between ECHDE and different Chaplygin gas forms including the modified variable Chaplygin gas (MVCG) and new modified Chaplygin gas (NMCG) and the viscous generalized Chaplygin gas (VGCG). In each case, we reconstruct the potentials and the dynamics of the scalar field which describe the entropy corrected holographic Chaplygin cosmology. The final section is devoted to the conclusion.

\section{THE MODEL}

We start by assuming the background spacetime to be spatially homogeneous and isotropic FRW spacetime given by

$$
d s^{2}=-d t^{2}+a^{2}(t)\left[\frac{d r^{2}}{1-k r^{2}}+r^{2}\left(d \theta^{2}+\sin ^{2} \theta d \varphi^{2}\right)\right] .
$$


Here $a(t)$ is the dimensionless scale factor which is an arbitrary function of time and $k$ is defined to be the curvature parameter which has dimensions of $l e n g t h^{-2}$ and its different values describe the spatial geometry. For instance, for $k=-1,0,1$, the above metric (2) represents the spatially open, flat and closed FRW spacetimes respectively. The Friedmann equation is given by

$$
H^{2}+\frac{k}{a^{2}}=\frac{1}{3 m_{p}^{2}}\left[\rho_{\Lambda}+\rho_{m}\right]
$$

where $m_{p}^{2}=(8 \pi G)^{-1}$ is modified Planck mass. Here $H=\dot{a} / a$ is the Hubble parameter while $\rho_{\Lambda}$ and $\rho_{m}$ are the energy densities of dark energy and matter respectively. In dimensionless form, Eq. (3) can be written as

$$
1+\Omega_{k}=\Omega_{\Lambda}+\Omega_{m}
$$

The dimensionless density parameters corresponding to matter, dark energy and curvature are

$$
\Omega_{m}=\frac{\rho_{m}}{\rho_{c r}}=\frac{\rho_{m}}{3 H^{2} m_{p}^{2}}, \Omega_{\Lambda}=\frac{\rho_{\Lambda}}{\rho_{c r}}=\frac{\rho_{\Lambda}}{3 H^{2} m_{p}^{2}}, \Omega_{k}=\frac{k}{(a H)^{2}} .
$$

Here $\rho_{c r}=3 H^{2} m_{p}^{2}$ is the critical density. The energy conservation equations for dark energy and dark matter are

$$
\begin{aligned}
\dot{\rho}_{\Lambda}+3 H\left(\rho_{\Lambda}+p_{\Lambda}\right) & =-Q \\
\dot{\rho}_{m}+3 H \rho_{m} & =Q .
\end{aligned}
$$

Here overdot represents the differentiation with respect to cosmic co-moving time $t$. Eq. (6) and (7) show that if there is an interaction between dark energy and dark matter, the energy conservation for dark energy and matter would not hold independently but for the total interacting system. In explicit form, we have $p_{\Lambda}=\omega_{\Lambda} \rho_{\Lambda}$ and $p_{m}=0$. During the energy transfer, local energy conservation will not hold in general but for the whole interacting system. Naturally if two species are present in dominant form, it is obvious that they will interact. If the quantity $Q$ is positive, it shows the transfer of energy from dark matter to dark energy and vice versa in the case when $Q$ is negative. The importance of interacting dark energy and dark matter model also emerges since it is the best fit for the data we obtain from the physical observations for instance SN Ia and cosmic microwave background [1]. 
Defining the effective equation of state for dark energy and dark matter as [13]

$$
\omega_{\Lambda}^{\mathrm{eff}}=\omega_{\Lambda}+\frac{\Gamma}{3 H}, \omega_{m}^{\mathrm{eff}}=-\frac{1}{r_{m}} \frac{\Gamma}{3 H},
$$

where the term $\Gamma=Q / \rho_{\Lambda}$ represents the decay rate. After employing Eq. (8) in (6) and (7), we obtain the following pair of continuity equations

$$
\begin{array}{r}
\dot{\rho}_{\Lambda}+3 H\left(1+\omega_{\Lambda}^{\mathrm{eff}}\right) \rho_{\Lambda}=0 \\
\dot{\rho}_{m}+3 H\left(1+\omega_{m}^{\mathrm{eff}}\right) \rho_{m}=0 .
\end{array}
$$

If we take $L$ as the Hubble scale $H^{-1}$ i.e. $L=H^{-1}$ at the present epoch $H=H_{0} \backsim 10^{-33} \mathrm{eV}$, then the energy density $\rho_{\Lambda}$ is comparable with the observed dark energy density $\backsim 10^{-10} \mathrm{eV}^{4}$. The second option for the infra-red cut-off is the particle horizon. Hsu [14] showed that under this scenario the resulting EoS becomes zero and does not lead to an accelerated universe. So to get an accelerated expansion of the observable universe, Li [15] proposed that the IR cut-off $L$ should be taken as the future event horizon and he defined it as

$$
L=a(t) r(t)
$$

here $r(t)$ is related to the future event horizon of the observable universe. Using the FRW metric, we can obtain [15]

$$
L=a(t) \frac{\operatorname{sinn}(\sqrt{|k|} y)}{\sqrt{|k|}}, \quad y=\frac{R_{h}}{a(t)},
$$

where $R_{h}$ is the size of the future event horizon defined as

$$
R_{h}=a(t) \int_{t}^{\infty} \frac{d t^{\prime}}{a\left(t^{\prime}\right)}=a(t) \int_{0}^{r_{1}} \frac{d r}{\sqrt{1-k r^{2}}} .
$$

The last integral has the explicit form as

$$
\int_{0}^{r_{1}} \frac{d r}{\sqrt{1-k r^{2}}}=\frac{1}{\sqrt{|k|}} \operatorname{sinn}^{-1}\left(\sqrt{|k|} r_{1}\right)= \begin{cases}\sin ^{-1}\left(r_{1}\right), & k=+1 \\ r_{1}, & k=0 \\ \sinh ^{-1}\left(r_{1}\right), & k=-1 .\end{cases}
$$

Using the definition of $\rho_{\Lambda}$ and $\rho_{c r}$, we obtain the following relation

$$
H L=\sqrt{\frac{3 n^{2} m_{p}^{2}+\gamma L^{-2} \ln \left(m_{p}^{2} L^{2}\right)+\beta L^{-2}}{3 m_{p}^{2} \Omega_{\Lambda}}} .
$$


Differentiate eq. (12) with respect to time $t$ and using Eq. (16), we have

$$
\dot{L}=\sqrt{\frac{3 n^{2} m_{p}^{2}+\gamma L^{-2} \ln \left(m_{p}^{2} L^{2}\right)+\beta L^{-2}}{3 m_{p}^{2} \Omega_{\Lambda}}}-\operatorname{cosn}(\sqrt{|k|} y),
$$

where

$$
\operatorname{cosn}(\sqrt{|k|} y)=\left\{\begin{array}{l}
\cos y, k=+1, \\
1, k=0, \\
\cosh y, k=-1,
\end{array}\right.
$$

After taking derivative of (1) with respect to $t$, we get

$$
\begin{aligned}
\dot{\rho}_{\Lambda}= & \left(2 \gamma L^{-5}-4 \gamma L^{-5} \ln \left(m_{p}^{2} L^{2}\right)-4 \beta L^{-5}-6 n^{2} m_{p}^{2} L^{-3}\right) \\
& \times\left[\sqrt{\frac{3 n^{2} m_{p}^{2}+\gamma L^{-2} \ln \left(m_{p}^{2} L^{2}\right)+\beta L^{-2}}{3 m_{p}^{2} \Omega_{\Lambda}}}-\operatorname{cosn}(\sqrt{|k|} y)\right] .
\end{aligned}
$$

Using Eq. (18) in (6), we arrive at

$$
\begin{aligned}
w_{\Lambda}= & -1-\left(\frac{2 \gamma L^{-2}-4 \gamma L^{-2} \ln \left(m_{p}^{2} L^{2}\right)-4 \beta L^{-2}-6 n^{2} m_{p}^{2}}{3\left(3 n^{2} m_{p}^{2}+\gamma L^{-2} \ln \left(m_{p}^{2} L^{2}\right)+\beta L^{-2}\right)}\right) \\
& \times\left[1-\sqrt{\frac{3 m_{p}^{2} \Omega_{\Lambda}}{3 n^{2} m_{p}^{2}+\gamma L^{-2} \ln \left(m_{p}^{2} L^{2}\right)+\beta L^{-2}}} \operatorname{cosn}(\sqrt{|k|} y)\right]-\frac{b^{2}\left(1+\Omega_{k}\right)}{\Omega_{\Lambda}} .
\end{aligned}
$$

Now making use of Eq. (19) in (8) yields

$$
\begin{aligned}
w_{\Lambda}^{\mathrm{eff}}= & -1-\left(\frac{2 \gamma L^{-2}-4 \gamma L^{-2} \ln \left(m_{p}^{2} L^{2}\right)-4 \beta L^{-2}-6 n^{2} m_{p}^{2}}{3\left(3 n^{2} m_{p}^{2}+\gamma L^{-2} \ln \left(m_{p}^{2} L^{2}\right)+\beta L^{-2}\right)}\right) \\
& \times\left[1-\sqrt{\frac{3 m_{p}^{2} \Omega_{\Lambda}}{3 n^{2} m_{p}^{2}+\gamma L^{-2} \ln \left(m_{p}^{2} L^{2}\right)+\beta L^{-2}}} \operatorname{cosn}(\sqrt{|k|} y)\right] .
\end{aligned}
$$

\section{CORRESPONDENCE BETWEEN ECHDE AND CHAPLYGIN GAS VARIANTS}

Since there are several candidates of dark energy, so it is essential to develop a correspondence and the relationships between them. Kamenshchik et al [16] studied a homogenous model based on a single fluid obeying the $\operatorname{EoS} p=-\frac{A_{0}}{\rho}$ called the Chaplygin gas, where $p$ and $\rho$ represent the pressure and energy density of the fluid and $A_{0}$ is some positive constant. Possessing many physically interesting features, several authors have used it to model the accelerated expansion of universe [17]. But it does not satisfactorily address the problems 
like structure formation and cosmological perturbation power spectrum [18]. Subsequently, this equation was modified to the form $p=-\frac{A_{0}}{\rho^{\alpha}}$ called generalized Chaplygin gas (GCG) to construct viable cosmological models. Two free parameters involved in it: one is $A_{0}$ and the other $0 \leq \alpha \leq 1$. The GCG fluid behaves like dust for small size of the universe while it acts as cosmological constant when universe gets sufficiently large. The GCG equation has been further modified to $p=B \rho-\frac{A_{0}}{\rho^{\alpha}}$ with $0 \leq \alpha \leq 1$, which is called modified Chaplygin gas (MCG) [19] and it involves three parameters. An interesting feature connected with MCG equation of state is that it shows radiations era in the early universe. At the late time it behaves as cosmological constant which can be fitted to a $\Lambda$ CDM model. Late on, Guo and Jhang [20] first proposed a model $p=B \rho-\frac{A_{0}}{\rho^{\alpha}}$ by taking $A_{0}$ as a function of the cosmological scale factor $a(t)$ i.e. $A_{0}=A_{0}(a(t))$, which is known as modified variable Chaplygin gas (MVCG) [21]. This assumption seems to be reasonable since $A_{0}(a(t))$ is related to scalar potential if we interpret Chaplygin gas via Born-Infeld scalar field.

\section{A. Modified variable Chaplygin gas and ECHDE}

Suppose we have two species i.e. dark matter and dark energy. The later is specified by the MVCG which is given by

$$
p=B \rho_{\Lambda}-\frac{B_{0} a^{-\delta}}{\rho_{\Lambda}^{\alpha}} .
$$

The evolution of the energy density of MVCG is

$$
\rho_{\Lambda}=\left[\frac{3(\alpha+1) B_{0}}{[3(\alpha+1)(B+1)-\delta]} \frac{1}{a^{\delta}}-\frac{C}{a^{3(\alpha+1)(B+1)}}\right]^{\frac{1}{\alpha+1}},
$$

where $B_{0}$ and $C$ are some constants.

We now reconstruct expressions of the potential and the dynamics of the scalar field in the presence of ECHDE. So for this, consider a time dependent scalar field $\phi(t)$ with potential $V(\phi)$, which are directly related with the energy density and pressure of MVCG as

$$
\begin{aligned}
& \rho_{\Lambda}=\frac{1}{2} \dot{\phi}^{2}+V(\phi), \\
& p_{\Lambda}=\frac{1}{2} \dot{\phi}^{2}-V(\phi) .
\end{aligned}
$$

Since the kinetic terms are positive hence it means that MVCG is of quintessence type. 
Adding Eqs. (23) and (24), we get the kinetic term

$$
\begin{aligned}
\dot{\phi}^{2}= & (1+B)\left[\frac{3(\alpha+1) B_{0}}{[3(\alpha+1)(B+1)-\delta]} \frac{1}{a^{\delta}}-\frac{C}{a^{3(\alpha+1)(B+1)}}\right]^{\frac{1}{\alpha+1}} \\
& -\frac{B_{0} a^{-\delta}}{\left[\frac{3(\alpha+1) B_{0}}{[3(\alpha+1)(B+1)-\delta]} \frac{1}{a^{\delta}}-\frac{C}{a^{3(\alpha+1)(B+1)}}\right]^{\frac{\alpha}{\alpha+1}}} .
\end{aligned}
$$

Subtraction of Eqs. (23) and (24) yields the potential term

$$
\begin{aligned}
2 V(\phi)= & (1-B)\left[\frac{3(\alpha+1) B_{0}}{[3(\alpha+1)(B+1)-\delta]} \frac{1}{a^{n}}-\frac{C}{a^{3(\alpha+1)(B+1)}}\right]^{\frac{1}{\alpha+1}} \\
& +\frac{B_{0} a^{-\delta}}{\left[\frac{3(\alpha+1) B_{0}}{[3(\alpha+1)(B+1)-\delta]} \frac{1}{a^{\delta}}-\frac{C}{a^{3(\alpha+1)(B+1)}}\right]^{\frac{\alpha}{\alpha+1}}} .
\end{aligned}
$$

To see the correspondence between the ECHDE and MVCG energy density, we use Eqs. (1) and (22) to get

$$
C=a^{3(\alpha+1)(B+1)}\left[\frac{3(\alpha+1) B_{0}}{[3(\alpha+1)(B+1)-\delta]} \frac{1}{a^{\delta}}-\left(3 n^{2} m_{p}^{2} L^{-2}+\gamma L^{-4} \ln \left(m_{p}^{2} L^{2}\right)+\beta L^{-4}\right)^{\alpha+1}\right] .
$$

Writing Eq. (21) in an alternate form

$$
B_{0}=a^{\delta}\left(B-w_{\Lambda}\right) \rho_{\Lambda}^{\alpha+1}
$$

Therefore in view of Eq. (19), the above equation (28) gives the value of the parameter $B_{0}$ as

$$
\begin{aligned}
B_{0}= & a^{\delta}\left(3 n^{2} m_{p}^{2} L^{-2}+\gamma L^{-4} \ln \left(m_{p}^{2} L^{2}\right)+\beta L^{-4}\right)^{\alpha+1}[1+B \\
& +\frac{2 \gamma L^{-2}-4 \gamma L^{-2} \ln \left(m_{p}^{2} L^{2}\right)-4 \beta L^{-2}-6 n^{2} m_{p}^{2}}{3\left(3 n^{2} m_{p}^{2}+\gamma L^{-2} \ln \left(m_{p}^{2} L^{2}\right)+\beta L^{-2}\right)} \\
& \left.\times\left(1-\sqrt{\frac{3 m_{p}^{2} \Omega_{\Lambda}}{3 n^{2} m_{p}^{2}+\gamma L^{-2} \ln \left(m_{p}^{2} L^{2}\right)+\beta L^{-2}}} \operatorname{cosn}(\sqrt{|k|} y)\right)+\frac{b^{2}\left(1+\Omega_{k}\right)}{\Omega_{\Lambda}}\right] .
\end{aligned}
$$

Now to determine the other parameter $C$ substitute the value of $B_{0}$ in Eq. (27), we have

$$
\begin{aligned}
C= & a^{3(\alpha+1)(A+1)}\left(3 n^{2} m_{p}^{2} L^{-2}+\gamma L^{-4} \ln \left(m_{p}^{2} L^{2}\right)+\beta L^{-4}\right)^{\alpha+1}\left(\frac{3(\alpha+1)}{[3(\alpha+1)(B+1)-\delta]}\right. \\
& \times\left(B+1+\frac{2 \gamma L^{-2}-4 \gamma L^{-2} \ln \left(m_{p}^{2} L^{2}\right)-4 \beta L^{-2}-6 n^{2} m_{p}^{2}}{3\left(3 n^{2} m_{p}^{2}+\gamma L^{-2} \ln \left(m_{p}^{2} L^{2}\right)+\beta L^{-2}\right)}\right) \\
& \left.\times\left[1-\sqrt{\frac{3 m_{p}^{2} \Omega_{\Lambda}}{3 n^{2} m_{p}^{2}+\gamma L^{-2} \ln \left(m_{p}^{2} L^{2}\right)+\beta L^{-2}}} \operatorname{cosn}(\sqrt{|k|} y)\right]-1\right)
\end{aligned}
$$


Now we can re-write the kinetic energy and scalar potential terms as

$$
\begin{aligned}
\dot{\phi}^{2}= & -\left(3 n^{2} m_{p}^{2} L^{-2}+\gamma L^{-4} \ln \left(m_{p}^{2} L^{2}\right)+\beta L^{-4}\right) \\
& \left(\frac{2 \gamma L^{-2}-4 \gamma L^{-2} \ln \left(m_{p}^{2} L^{2}\right)-4 \beta L^{-2}-6 n^{2} m_{p}^{2}}{3\left(3 n^{2} m_{p}^{2}+\gamma L^{-2} \ln \left(m_{p}^{2} L^{2}\right)+\beta L^{-2}\right)}\right. \\
& \left.\times\left[1-\sqrt{\frac{3 m_{p}^{2} \Omega_{\Lambda}}{3 n^{2} m_{p}^{2}+\gamma L^{-2} \ln \left(m_{p}^{2} L^{2}\right)+\beta L^{-2}}} \operatorname{cosn}(\sqrt{|k|} y)\right]+\frac{b^{2}\left(1+\Omega_{k}\right)}{\Omega_{\Lambda}}\right),
\end{aligned}
$$

and

$$
\begin{aligned}
2 V(\phi)= & \left(3 n^{2} m_{p}^{2} L^{-2}+\gamma L^{-4} \ln \left(m_{p}^{2} L^{2}\right)+\beta L^{-4}\right) \\
& \times\left(2+\frac{2 \gamma L^{-2}-4 \gamma L^{-2} \ln \left(m_{p}^{2} L^{2}\right)-4 \beta L^{-2}-6 n^{2} m_{p}^{2}}{3\left(3 n^{2} m_{p}^{2}+\gamma L^{-2} \ln \left(m_{p}^{2} L^{2}\right)+\beta L^{-2}\right)}\right. \\
& \left.\times\left[1-\sqrt{\frac{3 m_{p}^{2} \Omega_{\Lambda}}{3 n^{2} m_{p}^{2}+\gamma L^{-2} \ln \left(m_{p}^{2} L^{2}\right)+\beta L^{-2}}} \operatorname{cosn}(\sqrt{|k|} y)\right]+\frac{b^{2}\left(1+\Omega_{k}\right)}{\Omega_{\Lambda}}\right)
\end{aligned}
$$

Using the relation $x=\ln a$, we get $\dot{\phi}=\phi^{\prime} H$, where ' represents the derivative with respect to e-folding time parameter $\ln a$. After putting the value of $\dot{\phi}$ and applying integration, we obtain

$$
\begin{aligned}
\phi(a)-\phi\left(a_{0}\right)= & \frac{1}{H} \int_{0}^{\ln a}\left[-\left(3 n^{2} m_{p}^{2} L^{-2}+\gamma L^{-4} \ln \left(m_{p}^{2} L^{2}\right)+\beta L^{-4}\right)\right. \\
& \times\left(\frac{2 \gamma L^{-2}-4 \gamma L^{-2} \ln \left(m_{p}^{2} L^{2}\right)-4 \beta L^{-2}-6 n^{2} m_{p}^{2}}{3\left(3 n^{2} m_{p}^{2}+\gamma L^{-2} \ln \left(m_{p}^{2} L^{2}\right)+\beta L^{-2}\right)}\right. \\
& \left.\left.\times\left[1-\sqrt{\frac{3 m_{p}^{2} \Omega_{\Lambda}}{3 n^{2} m_{p}^{2}+\gamma L^{-2} \ln \left(m_{p}^{2} L^{2}\right)+\beta L^{-2}}} \operatorname{cosn}(\sqrt{|k|} y)\right]\right)+\frac{b^{2}\left(1+\Omega_{k}\right)}{\Omega_{\Lambda}}\right]^{1 / 2} d \ln a,
\end{aligned}
$$

where $a_{0}$ is the present value of the scale factor.

\section{B. New modified Chaplygin gas model and ECHDE}

The model that represents the dark energy is now the new modified Chaplygin gas (NMCG) given by [22]

$$
p_{\Lambda}=B \rho_{\Lambda}-\frac{K(a)}{\rho_{\Lambda}^{\alpha}}, \quad \text { where } B>0 \text { and } 0 \leq \alpha \leq 1
$$

Here $K(a)$ is a function of scale factor of the universe. Taking $K(a)$ in the form $K(a)=$ $-\omega_{\Lambda} A_{1} a^{-3\left(w_{\Lambda}+1\right)(\alpha+1)}$ as introduced by the authors in [23], we get

$$
p_{\Lambda}=B \rho_{\Lambda}+\frac{w_{\Lambda} A_{1}}{\rho_{\Lambda}^{\alpha}} a^{-3\left(w_{\Lambda}+1\right)(\alpha+1)} .
$$


The energy density of the NMCG can be expressed as

$$
\rho_{\Lambda}=\left[\frac{w_{\Lambda}}{w_{\Lambda}-B} A_{1} a^{-3\left(w_{\Lambda}+1\right)(\alpha+1)}+B_{1} a^{-3(B+1)(\alpha+1)}\right]^{\frac{1}{\alpha+1}}
$$

where $B_{1}$ is a constant of integration. Following the previous section we establish the correspondence between the ECHDE and NMCG energy density. Comparing Eqs. (36) and (1), we get

$$
\begin{aligned}
B_{1}= & a^{3(B+1)(\alpha+1)}\left(\left(3 n^{2} m_{p}^{2} L^{-2}+\gamma L^{-4} \ln \left(m_{p}^{2} L^{2}\right)+\beta L^{-4}\right)^{\alpha+1}\right. \\
& -\frac{1+\frac{2 \gamma L^{-2}-4 \gamma L^{-2} \ln \left(m_{p}^{2} L^{2}\right)-4 \beta L^{-2}-6 n^{2} m_{p}^{2}}{3\left(3 n^{2} m_{p}^{2}+\gamma L^{-2} \ln \left(m_{p}^{2} L^{2}\right)+\beta L^{-2}\right)} \times\left[1-\sqrt{\frac{3 m_{p}^{2} \Omega_{\Lambda}}{3 n^{2} m_{p}^{2}+\gamma L^{-2} \ln \left(m_{p}^{2} L^{2}\right)+\beta L^{-2}}} \operatorname{cosn}(\sqrt{|k|} y)\right]}{1+B+\frac{2 \gamma L^{-2}-4 \gamma L^{-2} \ln \left(m_{p}^{2} L^{2}\right)-4 \beta L^{-2}-6 n^{2} m_{p}^{2}}{3\left(3 n^{2} m_{p}^{2}+\gamma L^{-2} \ln \left(m_{p}^{2} L^{2}\right)+\beta L^{-2}\right)} \times\left[1-\sqrt{\frac{3 m_{p}^{2} \Omega_{\Lambda}}{3 n^{2} m_{p}^{2}+\gamma L^{-2} \ln \left(m_{p}^{2} L^{2}\right)+\beta L^{-2}}} \operatorname{cosn}(\sqrt{|k|} y)\right]} \\
& \left.\left.\times\left(A_{1} a^{3(\alpha+1) \frac{2 \gamma L^{-2}-4 \gamma L^{-2} \ln \left(m_{p}^{2} L^{2}\right)-4 \beta L^{-2}-6 n^{2} m_{p}^{2}}{3\left(3 n^{2} m_{p}^{2}+\gamma L^{-2} \ln \left(m_{p}^{2} L^{2}\right)+\beta L^{-2}\right)} \times\left[1-\sqrt{\frac{3 m_{p}^{2} \Omega_{\Lambda}}{3 n^{2} m_{p}^{2}+\gamma L^{-2} \ln \left(m_{p}^{2} L^{2}\right)+\beta L^{-2}}} \operatorname{cosn}(\sqrt{|k|} y)\right.}\right]\right)\right) .
\end{aligned}
$$

With the use of $p_{\Lambda}=\omega_{\Lambda} \rho_{\Lambda}$, Eq. (35) gives an expression for $A_{1}$ i.e.

$$
\begin{aligned}
A_{1}= & \left(3 n^{2} m_{p}^{2} L^{-2}+\gamma L^{-4} \ln \left(m_{p}^{2} L^{2}\right)+\beta L^{-4}\right)^{\alpha+1} \\
& \times a \\
& \times\left[\frac{B(\alpha+1) \frac{2 \gamma L^{-2}-4 \gamma L^{-2} \ln \left(m_{p}^{2} L^{2}\right)-4 \beta L^{-2}-6 n^{2} m_{p}^{2}}{3\left(3 n^{2} m_{p}^{2}+\gamma L^{-2} \ln \left(m_{p}^{2} L^{2}\right)+\beta L^{-2}\right)} \times\left(1-\sqrt{\frac{3 m_{p}^{2} \Omega_{\Lambda}}{3 n^{2} m_{p}^{2}+\gamma L^{-2} \ln \left(m_{p}^{2} L^{2}\right)+\beta L^{-2}}} \operatorname{cosn}(\sqrt{|k| y)})\right.}{1+\frac{2 \gamma L^{-2}-4 \gamma L^{-2} \ln \left(m_{p}^{2} L^{2}\right)-4 \beta L^{-2}-6 n^{2} m_{p}^{2}}{3\left(3 n^{2} m_{p}^{2}+\gamma L^{-2} \ln \left(m_{p}^{2} L^{2}\right)+\beta L^{-2}\right)} \times\left[1-\sqrt{\frac{3 m_{p}^{2} \Omega_{\Lambda}}{3 n^{2} m_{p}^{2}+\gamma L^{-2} \ln \left(m_{p}^{2} L^{2}\right)+\beta L^{-2}}} \operatorname{cosn}(\sqrt{|k|} y)\right]}\right]
\end{aligned}
$$

So we have found the expressions for the constants $A_{1}$ and $B_{1}$. Employing Eqs. (23) and (24), the kinetic and potential terms are found to be

$$
\begin{aligned}
\dot{\phi}^{2}= & (B+1)\left[\frac{w_{\Lambda}}{w_{\Lambda}-B} A_{1} a^{-3\left(w_{\Lambda}+1\right)(\alpha+1)}+B_{1} a^{-3(B+1)(\alpha+1)}\right]^{\frac{1}{\alpha+1}} \\
& +\frac{A_{1} w_{\Lambda} a^{-3\left(w_{\Lambda}+1\right)(\alpha+1)}}{\left[\frac{w_{\Lambda}}{w_{\Lambda}-B} A_{1} a^{-3\left(w_{\Lambda}+1\right)(\alpha+1)}+B_{1} a^{-3(B+1)(\alpha+1)}\right]^{\frac{\alpha}{\alpha+1}}},
\end{aligned}
$$

and

$$
\begin{aligned}
2 V(\phi)= & (1-B)\left[\frac{w_{\Lambda}}{w_{\Lambda}-B} A_{1} a^{-3\left(w_{\Lambda}+1\right)(\alpha+1)}+B_{1} a^{-3(B+1)(\alpha+1)}\right]^{\frac{1}{\alpha+1}} \\
& -\frac{A_{1} w_{\Lambda} a^{-3\left(w_{\Lambda}+1\right)(\alpha+1)}}{\left[\frac{w_{\Lambda}}{w_{\Lambda}-B} A_{1} a^{-3\left(w_{\Lambda}+1\right)(\alpha+1)}+B_{1} a^{-3(B+1)(\alpha+1)}\right]^{\frac{\alpha}{\alpha+1}}},
\end{aligned}
$$

where $A_{1}$ and $B_{1}$ are given in (38) and (37) respectively. In all Eqs. (35), (36), (39) and (40) the value of $w_{\Lambda}$ is given by Eq. (19). From Eqs. (23) and (24), the kinetic energy term 
is re-written to be

$$
\begin{aligned}
\dot{\phi}^{2}= & -\left(3 n^{2} m_{p}^{2} L^{-2}+\gamma L^{-4} \ln \left(m_{p}^{2} L^{2}\right)+\beta L^{-4}\right) \\
& \times\left(\frac{2 \gamma L^{-2}-4 \gamma L^{-2} \ln \left(m_{p}^{2} L^{2}\right)-4 \beta L^{-2}-6 n^{2} m_{p}^{2}}{3\left(3 n^{2} m_{p}^{2}+\gamma L^{-2} \ln \left(m_{p}^{2} L^{2}\right)+\beta L^{-2}\right)}\right. \\
& \left.\times\left[1-\sqrt{\frac{3 m_{p}^{2} \Omega_{\Lambda}}{3 n^{2} m_{p}^{2}+\gamma L^{-2} \ln \left(m_{p}^{2} L^{2}\right)+\beta L^{-2}}} \operatorname{cosn}(\sqrt{|k|} y)\right]+\frac{b^{2}\left(1+\Omega_{k}\right)}{\Omega_{\Lambda}}\right),
\end{aligned}
$$

while the potential energy term has the form

$$
\begin{aligned}
2 V(\phi)= & \left(3 n^{2} m_{p}^{2} L^{-2}+\gamma L^{-4} \ln \left(m_{p}^{2} L^{2}\right)+\beta L^{-4}\right) \\
& \times\left(2+\frac{2 \gamma L^{-2}-4 \gamma L^{-2} \ln \left(m_{p}^{2} L^{2}\right)-4 \beta L^{-2}-6 n^{2} m_{p}^{2}}{3\left(3 n^{2} m_{p}^{2}+\gamma L^{-2} \ln \left(m_{p}^{2} L^{2}\right)+\beta L^{-2}\right)}\right. \\
& \left.\times\left[1-\sqrt{\frac{3 m_{p}^{2} \Omega_{\Lambda}}{3 n^{2} m_{p}^{2}+\gamma L^{-2} \ln \left(m_{p}^{2} L^{2}\right)+\beta L^{-2}}} \operatorname{cosn}(\sqrt{|k|} y)\right]+\frac{b^{2}\left(1+\Omega_{k}\right)}{\Omega_{\Lambda}}\right) .
\end{aligned}
$$

Following the same steps as done for the MVCG, the kinetic term is easily transformable to the following form

$$
\begin{aligned}
\phi(a)-\phi\left(a_{0}\right)= & \frac{1}{H} \int_{0}^{\ln a}\left[-\left(3 n^{2} m_{p}^{2} L^{-2}+\gamma L^{-4} \ln \left(m_{p}^{2} L^{2}\right)+\beta L^{-4}\right)\right. \\
& \times \frac{2 \gamma L^{-2}-4 \gamma L^{-2} \ln \left(m_{p}^{2} L^{2}\right)-4 \beta L^{-2}-6 n^{2} m_{p}^{2}}{3\left(3 n^{2} m_{p}^{2}+\gamma L^{-2} \ln \left(m_{p}^{2} L^{2}\right)+\beta L^{-2}\right)} \\
& \left.\times\left[1-\sqrt{\frac{3 m_{p}^{2} \Omega_{\Lambda}}{3 n^{2} m_{p}^{2}+\gamma L^{-2} \ln \left(m_{p}^{2} L^{2}\right)+\beta L^{-2}}} \operatorname{cosn}(\sqrt{|k|} y)\right]+\frac{b^{2}\left(1+\Omega_{k}\right)}{\Omega_{\Lambda}}\right]^{1 / 2} d \ln a .
\end{aligned}
$$

\section{Viscous generalized Chaplygin gas model and ECHDE}

If we assume that the dark energy as non-viscous as taken in section $\mathrm{A}$ and $\mathrm{B}$, it must results in the occurrence of a cosmic singularity (Big Rip) in the far future. This singularity can be alleviated by introducing the quantum corrections due to the conformal anomaly while the other option is to consider the bulk viscosity $\xi$ of the cosmic fluid [24]. The theory of bulk viscosity was initially investigated by Eckart and later on pursued by Landau and Lifshitz [25]. The important feature of dark energy with bulk viscosity is that it shows the accelerated expansion of phantom type in the later epoch and softens the coincidence 
problem, age problem and phantom crossing [26]. The effective pressure containing the isotropic pressure and viscous stress is given by the equation of state

$$
p_{\text {eff }}=p_{\Lambda}+\Pi \text {, }
$$

where $p_{\Lambda}=\frac{\chi}{\rho_{\Lambda}^{\alpha}}, \chi>0$. Notice that first term on the right hand side mimics the GCG and the parameter $\alpha$ varies as $0<\alpha \leq 1$. If $\alpha=1$ it represents the Chaplygin gas model. On the other hand if $\alpha<0$, it corresponds to a polytropic gas. The bulk viscous fluid is represented by $\Pi=-\xi\left(\rho_{\Lambda}\right) u_{; \mu}^{\mu}$ where $u^{\mu}$ is the four-velocity vector of the viscous fluid and $\xi>0$ to get the positive entropy production in conformity with second law of thermodynamics [27]. We choose $\xi\left(\rho_{\Lambda}\right)=\nu \rho_{\Lambda}^{1 / 2}$, with $\nu$ as constant. The energy conservation equation yields the energy density of VGCG as

$$
\rho_{\Lambda}=\left[\frac{D a^{-3(\alpha+1)\left(1-\nu \gamma_{1}\right)}-\chi}{\left(1-\nu \gamma_{1}\right)}\right]^{\frac{1}{\alpha+1}}
$$

[28]. Here $\gamma_{1}=m_{p}^{-1} \sqrt{1-r_{m}}$, where $r_{m}=\frac{\rho_{m}}{\rho_{\Lambda}}=\frac{\Omega_{m}}{\Omega_{\Lambda}}$ and $D$ some constant of integration. The effective equation of state (45) says that the universe is expanding in accelerated manner. It has been shown that this phenomenon of universe can be studied with the help of dynamical evolving scalar fields usually called inflation. So it was introduced to construct models dealing with minimally coupled scalar field. Hence we construct the dynamical scalar field $\phi$ with potential $V(\phi)$, related to the energy density and pressure of viscous dark energy model as

$$
\begin{aligned}
\rho_{\Lambda} & =\left[\frac{D a^{-3(\alpha+1)\left(1-\nu \gamma_{1}\right)}-\chi}{\left(1-\nu \gamma_{1}\right)}\right]^{\frac{1}{\alpha+1}} \\
p_{\Lambda} & =\chi\left[\frac{\left(1-\nu \gamma_{1}\right)}{D a^{-3(\alpha+1)\left(1-\nu \gamma_{1}\right)}-\chi}\right]^{\frac{\alpha}{\alpha+1}}-3 \nu H\left[\frac{D a^{-3(\alpha+1)\left(1-\nu \gamma_{1}\right)}-\chi}{\left(1-\nu \gamma_{1}\right)}\right]^{1 / 2} .
\end{aligned}
$$

To determine the expressions for the unknown $D$ and $\chi$ we take the following steps. The effective equation of state for the interacting VGCG is

$$
w_{\Lambda}^{\mathrm{eff}}=\frac{\chi}{\rho_{\Lambda}^{\alpha+1}}-3 v H \rho_{\Lambda}^{-\frac{1}{2}}+\frac{b^{2}\left(1+\Omega_{k}\right)}{\Omega_{\Lambda}} .
$$


After substituting the value of $w_{\Lambda}^{\text {eff }}$ in (47) and making simplification, we obtain

$$
\begin{aligned}
\chi= & \left(3 n^{2} m_{p}^{2} L^{-2}+\gamma L^{-4} \ln \left(m_{p}^{2} L^{2}\right)+\beta L^{-4}\right)^{\alpha+1} \\
& \times\left(-1-\frac{2 \gamma L^{-2}-4 \gamma L^{-2} \ln \left(m_{p}^{2} L^{2}\right)-4 \beta L^{-2}-6 n^{2} m_{p}^{2}}{3\left(3 n^{2} m_{p}^{2}+\gamma L^{-2} \ln \left(m_{p}^{2} L^{2}\right)+\beta L^{-2}\right)}\right. \\
& \times\left[1-\sqrt{\frac{3 m_{p}^{2} \Omega_{\Lambda}}{3 n^{2} m_{p}^{2}+\gamma L^{-2} \ln \left(m_{p}^{2} L^{2}\right)+\beta L^{-2}}} \operatorname{cosn}(\sqrt{|k|} y)\right] \\
& \left.+3 v H\left(3 n^{2} m_{p}^{2} L^{-2}+\gamma L^{-4} \ln \left(m_{p}^{2} L^{2}\right)+\beta L^{-4}\right)^{-1 / 2}-\frac{b^{2}\left(1+\Omega_{k}\right)}{\Omega_{\Lambda}}\right) .
\end{aligned}
$$

Inserting the value of $\chi$ in (45), we get

$$
\begin{aligned}
D= & a^{3(\alpha+1)\left(1-\nu \gamma_{1}\right)}\left(3 n^{2} m_{p}^{2} L^{-2}+\gamma L^{-4} \ln \left(m_{p}^{2} L^{2}\right)+\beta L^{-4}\right)^{\alpha+1} \\
& \times\left[-\nu \gamma_{1}-\frac{2 \gamma L^{-2}-4 \gamma L^{-2} \ln \left(m_{p}^{2} L^{2}\right)-4 \beta L^{-2}-6 n^{2} m_{p}^{2}}{3\left(3 n^{2} m_{p}^{2}+\gamma L^{-2} \ln \left(m_{p}^{2} L^{2}\right)+\beta L^{-2}\right)}\right. \\
& \times\left[1-\sqrt{\frac{3 m_{p}^{2} \Omega_{\Lambda}}{3 n^{2} m_{p}^{2}+\gamma L^{-2} \ln \left(m_{p}^{2} L^{2}\right)+\beta L^{-2}}} \operatorname{cosn}(\sqrt{|k|} y)\right] \\
& \left.+3 v H\left(3 n^{2} m_{p}^{2} L^{-2}+\gamma L^{-4} \ln \left(m_{p}^{2} L^{2}\right)+\beta L^{-4}\right)^{-\frac{1}{2}}-\frac{b^{2}\left(1+\Omega_{k}\right)}{\Omega_{\Lambda}}\right] .
\end{aligned}
$$

Now we can re-write the scalar potential and kinetic energy terms as following

$$
\begin{aligned}
2 V(\phi)= & \left(3 n^{2} m_{p}^{2} L^{-2}+\gamma L^{-4} \ln \left(m_{p}^{2} L^{2}\right)+\beta L^{-4}\right) \\
& \times\left(2+\frac{2 \gamma L^{-2}-4 \gamma L^{-2} \ln \left(m_{p}^{2} L^{2}\right)-4 \beta L^{-2}-6 n^{2} m_{p}^{2}}{3\left(3 n^{2} m_{p}^{2}+\gamma L^{-2} \ln \left(m_{p}^{2} L^{2}\right)+\beta L^{-2}\right)}\right. \\
& \left.\times\left[1-\sqrt{\frac{3 m_{p}^{2} \Omega_{\Lambda}}{3 n^{2} m_{p}^{2}+\gamma L^{-2} \ln \left(m_{p}^{2} L^{2}\right)+\beta L^{-2}}} \operatorname{cosn}(\sqrt{|k|} y)\right]+\frac{b^{2}\left(1+\Omega_{k}\right)}{\Omega_{\Lambda}}\right) .
\end{aligned}
$$

and

$$
\begin{aligned}
\dot{\phi}^{2}= & -\left(3 n^{2} m_{p}^{2} L^{-2}+\gamma L^{-4} \ln \left(m_{p}^{2} L^{2}\right)+\beta L^{-4}\right) \\
& \times\left(\frac{2 \gamma L^{-2}-4 \gamma L^{-2} \ln \left(m_{p}^{2} L^{2}\right)-4 \beta L^{-2}-6 n^{2} m_{p}^{2}}{3\left(3 n^{2} m_{p}^{2}+\gamma L^{-2} \ln \left(m_{p}^{2} L^{2}\right)+\beta L^{-2}\right)}\right. \\
& \left.\times\left[1-\sqrt{\frac{3 m_{p}^{2} \Omega_{\Lambda}}{3 n^{2} m_{p}^{2}+\gamma L^{-2} \ln \left(m_{p}^{2} L^{2}\right)+\beta L^{-2}}} \operatorname{cosn}(\sqrt{|k|} y)\right]+\frac{b^{2}\left(1+\Omega_{k}\right)}{\Omega_{\Lambda}}\right) .
\end{aligned}
$$


Again using the equation $\dot{\phi}=\phi^{\prime} H$, we can write

$$
\begin{aligned}
\phi(a)-\phi\left(a_{0}\right)= & \frac{1}{H} \int_{0}^{\ln a}\left[-\left(3 n^{2} m_{p}^{2} L^{-2}+\gamma L^{-4} \ln \left(m_{p}^{2} L^{2}\right)+\beta L^{-4}\right)\right. \\
& \times\left(\frac{2 \gamma L^{-2}-4 \gamma L^{-2} \ln \left(m_{p}^{2} L^{2}\right)-4 \beta L^{-2}-6 n^{2} m_{p}^{2}}{3\left(3 n^{2} m_{p}^{2}+\gamma L^{-2} \ln \left(m_{p}^{2} L^{2}\right)+\beta L^{-2}\right)}\right. \\
& \left.\left.\times\left[1-\sqrt{\frac{3 m_{p}^{2} \Omega_{\Lambda}}{3 n^{2} m_{p}^{2}+\gamma L^{-2} \ln \left(m_{p}^{2} L^{2}\right)+\beta L^{-2}}} \cos (\sqrt{|k|} y)\right]+\frac{b^{2}\left(1+\Omega_{k}\right)}{\Omega_{\Lambda}}\right)\right]^{1 / 2} d \ln a .
\end{aligned}
$$

It is interesting to note that the above potential and kinetic energy expressions for the interacting ECHDE with the viscous generalized Chaplygin gas coincide with the non-viscous case.

\section{CONCLUSION}

Enormous literature dealing with the subject of dark energy is available but the holographic dark energy is considered to be the most promising candidate of dark energy. In this paper we have constructed a correspondence between the interacting ECHDE and the Chaplygin gas variants. Several candidates of dark energy have been suggested to describe cosmic acceleration but Chaplygin gas has emerged as a unification of dark energy and dark matter. It's cosmic evolution is similar to initial dust like matter while it behaves as a cosmological constant at a later epoch. In the present work, we have investigated a model of dark energy in the presence of entropy corrections to holographic dark energy. In this context, a link between the ECHDE and various models of Chaplygin gas has been established. We have found the kinetic and potential energies corresponding to each model and also reconstructed the potentials.

[1] A. G.Riess et al. [Supernova Search Team Collaboration], Astron. J. 116 (1998)1009; S. perlmutter et al., [Supernova Cosmology Project Collaboration], Astrophys. J. 517 (1999) 565; W. J. Percival et al., [The 2d FGRS Collaboration], Mon .Not. Roy. Astron. Soc. 327 (2001) 1297; P. Astier et al., Astron., Astrophs. 447 (2006) 31; A. G. Riess et al., [Supernova Search Team Collaboration], Astrophys. J. 607 (2004) 665; P. de Bernardis et al., Nature 
(London) 404 (2000) 955; R. A Knop et al., Astrophys. J. 598 (2003) 102; J. L. Tonry et al., Astrophys. J. 594 (2003) 1; M. V. Jhon Astrophys. J. 614 (2004) 1; D. N. Spergel et al., Astrophys. J. Suppl. 170 (2007) 377; G. Hinshaw Astrophys. J. 170 (2007) 288; M. Colless et al., Mon. Not. R. Astro. Soc. 328 (2001) 1039; M. Tegmark et al., Phys Rev. D 69 (2004) 103501; V. Springel et al., Nature (London) 440 (2006) 1137.

[2] P. J. E. Peebles and B. Ratra, Rev. Mod. Phys. 75 (2003) 559; E. J. Copeland et al., Int. J. Mod. Phys. D 15 (2006) 1753; R. R. Caldwell et al., Phys. Rev. Lett. 80, (1998) 1582; A. R. Liddle et al., Phys. Rev. D 59 (1999) 023509; T. padmanabhan 380 (2003) 235; A. Sen, JHEP 0207, (2002) 065; N.N. Weinberg et al., Phys. Rev. Lett. 91, (2003) 071301 ; B. Feng et al., Phys. Lett B 607 (2005) 35; Z. K. Guo et al., Phys. Lett. B 608 (2005) 177; H. Wei et al., Class. Quantum Grav. 22 (2005) 3189; S. M. Carrol. The Cosmological Constant, Living Rev. Rel. 4 (2001) 1; V Sahni et al., Int. J. Mod. Phys. D 9 (2000) 373.

[3] H. Zhang et al., Phys.Lett. B 678 (2009) 331; G. Caldera-Cabral et al.,JCAP 07 (2009) 027; M. Jamil et al., Eur. Phys. J. C 64 (2009) 97; M. Jamil et al., Eur. Phys. J. C 60 (2009) 141; M. Jamil et al., Eur. Phys. J. C 58 (2008) 111.

[4] Sadjadi H. M. et al., Phys. Rev. D 74 (2006) 103007.

[5] N. J. Poplawski Phys. Rev. D 74 (2006) 084032; A. Sheykhi Phys. Lett. B 681 (2009) 205; K. Y. Kim et al., Mod. Phys. Lett. A 22 (2007) 2631 M. R. Setare arXiv: 0909.0456; M. R. Setare Chin. Phys. Lett. 26 (2009) 029501.

[6] K. Enqvist, S et al., JCAP 2 (2005) 004; X. Zhang. Int. J. Mod. Phys. D 14 (2005) 1597; D. Pavon et al., hep-th/0511053; ; P. F. Gonzalez-Diaz, Phys. Rev. D 27 (1983) 3042.

[7] G’t Hooft, gr-qc/9310026; L. Susskind, J. Math. Phys. 36 (1995) 6377.

[8] J. D. Bekenstein, Phys. Rev. D 7 (1973) 2333; S. W. Hawking Comm. Math. Phys. 43 (1975) 199; S. W. Hawking Phys. Rev. D 13 (1976) 191; J. D. bekenstein, Phys. Rev. D 23 (1981) 287; A. G. Cohen et al., Phys. Rev. Lett. 82 (1999) 4971.

[9] M. R. Setare Eur. Phys. J. C 50 (2007) 991; M. R. Setare Eur. Phys. J. C 52 (2007) 689; K. Karami et al., arXiv: 0912.1536.

[10] H. Wei, Commun. Theor. Phys. 52 (2009) 743; M. Jamil and M.U. Farooq, JCAP 1003 (2010) 001.

[11] A. Ghosh and P. Mitra, Phys. Rev. D 71 (2005) 027502; A. Ashtekar et al, Phys. Rev. Lett. 80 (1998) 904; C. Rovelli, Phys. Rev. Lett. 77 (1996) 3288; K. A. Miessner, Class. Quant. 
Grav. 21 (2004) 5245.

[12] L. Xu JCAP 09 (2009) 016.

[13] H. Kim et al., Phys. Lett. B 632 (2006) 605; H. M. Sadjadi et al., Phys. Rev. D 74 (2006) 103007.

[14] S. D. H. Hsu, Phys. Lett. B 594 (2004) 13.

[15] M. Li, Phys. Lett. B 603 (2004) 1.

[16] A. Kamenshchik et al., Phys. Lett. B 511 (2001) 265.

[17] V. Gorini et al., preprint 0403062 [gr-qc]; A. Y. Kamenshchik et al., Phys. Lett. B 511 (2001) 265.

[18] Sandvik et al., Phys. Rev. D 69 (2004) 123524; R. Bean R et al., Phys. Rev. D 68 (2003) 023515 .

[19] H. B. Benaoum, preprint hep-th/0205140 (2002); ;U. Debnath et al., Class.Qunatum Grav. 21 (2004) 5609; V. Gorini et al., Phys. Rev. D 67 (2003) 063509; U. Alam et al., Mon. Not. Roy. Astrn. Soc. 344 (2003) 1057; A. Dev et al., Phys Rev D 67 (2003) 023515. V. Sahni et al JETP Lett. 77 (2003) 201; M. C. Bento, et al., Phys. Rev. D 66 (2002) 043507.

[20] Z. K. Guo and Y. Z. Zhang, Phys. Lett. B 645 (2007) 326.

[21] U. Debnath, Astrophys. Space Sci., 312 (2007) 295; M. Jamil et al., Eur. J. C 61 (2009) 471.

[22] Surajit Chattopadhyay, Ujjal Debnath, arXiv.: 0805.007v [gr-qc].

[23] X. Zhang et al., JCAP 01 (2006) 003.

[24] I. Brevik, S. D. Odintsov, Phys. Lett. B 455 (1999) 104; S. Nojiri, S.D. Odintsov Phys. Lett. B 562 (2003) 147.

[25] C. Eckart, Phys. Rev. 58 (1940) 919; L. D. Landau and E. M. Lifschitz, Fluid Mechanics (Butterworth Heineman, Oxford (1987))

[26] C. Feng et al., Phys Lett. B 680 (2009) 355; J. Chen, Y. Wang. :arXiv: 0904.2808v2 [gr,qc]; I. Brevik, Int. J. Mod. Phys. D 15, (2006) 767.

[27] W. Zimdahl, D. Pavon, Int. J. Mod. Phys. D 15 (2006) 767.

[28] M. Jamil, M. A. Rashid, Eur. Phys. J. C 56 (2008) 429. 